\title{
A influência da escolaridade na percepção sobre alimentos considerados saudáveis
}

Andréia Ferreira de Moura1, Deborah Cristina Landi Masquio²

\section{Resumo}

O presente trabalho objetiva identificar os alimentos considerados indispensáveis à alimentação saudável pela população carente, segundo a escolaridade. Trata-se de estudo transversal realizado em instituições filantrópicas do município de Mauá, SP. A pesquisa consistiu em atividade dinâmica, na qual foram disponibilizadas figuras de alimentos. Os voluntários foram orientados a colar em folha sulfite os produtos que consideravam indispensáveis à alimentação saudável. Participaram da pesquisa 136 voluntários de diversas faixas etárias. Os alimentos mais citados como sendo indispensáveis pela amostra total do estudo foram o grupo das frutas, verduras e legumes (99\%), carnes (87\%), arroz $(81 \%)$, leite $(80 \%)$, feijão (78\%), iogurte $(70 \%)$, ovo $(69 \%)$ e gelatina (67\%). Observou-se associação significativa entre a escolaridade e a citação de alguns alimentos como suco artificial $(p=0,00)$, embutidos $(p=0,00)$, empanado de frango $(p=0,01)$, cereal matinal $(p=0,04)$, biscoito recheado $(p=0,01)$, sopa instantânea $(p=0,00)$, maionese $(p=0,00)$, extrato de tomate $(p=0,01)$, macarrão instantâneo $(p=0,02)$ e temperos prontos $(p=0,01)$. Na categoria de pessoas que não estudaram notou-se maior percepção do suco artificial (75\%), embutidos $(58,3 \%)$, maionese $(66,7 \%)$ e macarrão instantâneo (58,3\%) como alimentos saudáveis. De maneira inversa, a categoria de pessoas que completaram o ensino médio apresentou menor prevalência na citação de suco artificial (25\%), embutidos (10,7\%), empanado de frango (0\%), maionese $(7,1 \%)$, extrato de tomate $(10,7 \%)$ e temperos prontos $(3,6 \%)$ como alimentos saudáveis. Tais dados revelam associação significativa sobre a consideração de alimentos processados como sendo indispensáveis à alimentação saudável e o nível de escolaridade. Os resultados sugerem que a população menos escolarizada pode estar mais suscetível a interpretações errôneas sobre conceitos de alimentação e nutrição.

\section{Palavras-chave}

Escolaridade. Educação Nutricional. Comportamento Alimentar.

1. Mestranda no Programa de Pós-Graduação em Cardiologia da Universidade Federal de São Paulo, nutricionista com especialização em Obesidade, Emagrecimento e Saúde: Abordagem Multidisciplinar pela Universidade Federal de São Paulo.E-mail: andreiamoura_nutri@yahoo.com.br.

2. Doutoranda em Ciências no Programa dé Pós-Graduação em Nutrição da Universidade Federal de São Paulo, nutricionista. E-mail: deborah.masquio@unifesp.br. 


\title{
The influence of education on people's view about healthy food
}

Andréia Ferreira de Moura*, Deborah Cristina Landi Masquio**

\begin{abstract}
This study aimed to identify the foods considered essential to a healthy eating by needy population, according to their education level. It is a cross-sectional study that was conducted in a philanthropic institution in Mauá, São Paulo state. The research consisted of a dynamic activity in which food images were available. The volunteers were told to stick on a sheet paper those products they considered necessary for a healthy diet. 136 volunteers of different age groups participated in the research. Foods that chosen by the participants were: fruits and vegetables group (99\%), meats $(87 \%)$, rice $(81 \%)$, milk $(80 \%)$, beans $(78 \%)$, yogurt $(70 \%)$, egg $(69 \%)$ and gelatin $(67 \%)$. The results showed a significant association between the level of education and some foods choices, such as artificial juice $(p=0.00)$, cold cuts $(p=0.00)$, breaded chicken $(p=0.01)$, breakfast cereal $(p=0.04)$, sandwich biscuit $(p=0.01)$, instant soup $(p=0.00)$, mayonnaise $(p=0.00)$, tomato paste $(p=0.01)$, instant noodles $(p=0.02)$ and ready-made seasoning $(p=0.01)$. On the one hand, in people who did not study category, there was a high prevalence of choices of artificial juice (75\%), cold cuts $(58.3 \%)$, mayonnaise $(66.7 \%)$ and instant noodles $(58.3 \%)$ as healthy foods. On the other hand, in the people who have completed high school category, there was a low prevalence of choice of artificial juice $(25 \%)$, sausages $(10.7 \%)$, breaded chicken $(0 \%)$, mayonnaise $(7.1 \%)$, tomato paste $(10.7 \%)$ and ready-made seasoning $(3.6 \%)$ as healthy foods. These data show the significant association between the consideration of processed foods as healthy foods and levels of education. The results suggest that less educated people may be more susceptible to misconceptions about diet and nutrition.
\end{abstract}

\section{Keywords}

Educational Status. Food and Nutrition Education. Eating Behavior.

\footnotetext{
* Master's student in the Cardiology Postgraduate Program of the Federal University of São Paulo and nutritionist specialized in Obesity, Weight loss and Health: Multidisciplinary Approach from the Federal University of São Paulo. E-mail: andreinamoura_unitri.com.br.

* Doctoral student in the Postgraduate Program in Nutrition at the Federal University of São Paulo. E-mail: deborah.masquito@unifesp.br.
} 


\section{Introdução}

A prevalência da obesidade na população brasileira está aumentando de forma crescente e alguns dos fatores que contribuem para ascensão desta epidemia é a transição nutricional (WANG; MONTEIRO; POPKIN, 2002). A característica básica dessa transição é o crescimento da dieta rica em gorduras, açúcares, alimentos refinados e a redução de carboidratos complexos e fibras. As refeições rápidas e fora do lar com refrigerantes, salgadinhos, sanduíches e biscoitos substituíram o arroz, o feijão, a carne e as frutas e hortaliças (TARDIDO; FALCÃO, 2006).

O nível de escolaridade tem sido apontado como variável capaz de interferir na forma como a população escolhe seus alimentos, que pode ser decisiva para a qualidade do autocuidado e para a capacidade de interpretar informações relativas à proteção da saúde (VELÁSQUEZMELÉNDEZ; PIMENTA; KAC, 2004). Desta forma, acredita-se que a educação escolar é capaz de influenciar o conhecimento sobre alimentação e nutrição (CASTRO; DÁTILLO; LOPES, 2010) que, por sua vez, relaciona-se ao estado nutricional dos indivíduos (DÁTILLO et al., 2009). Observa-se que o baixo grau de conhecimento em nutrição está associado aos desvios nutricionais em populações de baixa renda (BRITO etal., 2012). Dados do ano de 2012 revelam excesso de peso de $57,3 \%$ na população brasileira maior de 18 anos que estudou por no máximo oito anos. Essa porcentagem reduz para $48,4 \%$ na categoria de pessoas que estudaram 12 anos ou mais. A diferença dos dados é ainda maior entre a população feminina. A prevalência de excesso de peso em mulheres com baixo nível de escolaridade é de 57,4\%, enquanto que, para aquelas que estudaram 12 anos ou mais, é de 40,2\% (BRASIL, 2012).

Sabe-se que esse público é mais suscetível a "tabus alimentares" o que, juntamente com a falta de informação adequada, pode agravar seu estado de saúde (BRITO et al., 2012). Nesse sentido, é importante destacar que a escolaridade foi apontada como a variável chave que responde pela associação inversa encontrada no Brasil entre nível socioeconômico e obesidade em mulheres (MONTEIRO; CONDE; POPKIN, 2001).

Demais estudos têm sugerido que a baixa escolaridade pode ser fator de risco para o sobrepeso e a obesidade (WARDLE; PARMENTER; WALLER, 2000; WARDLE; WALLER; JARVIS, 2002; MACHADO; SICHIERI, 2002; GIUGLIANO; CARNEIRO, 2004; MONTEIRO; CONDE; CASTRO, 2003; MOLARIUS et al., 2000; TEICHMANN et al. 2006; GUTIERREZ-FISAC et al., 2002; TORRANCE; HOPER; REEDER, 2002; VELÁSQUEZ-MELÉNDEZ; PIMENTA; KAC, 2004). Wardle e outros (2002) verificaram que homens e mulheres que abandonam a escola estão mais propensos à obesidade que aqueles/aquelas com mais tempo de estudo, sendo que a escolaridade foi mais associada ao estado nutricional do que fatores como renda e ocupação. Machado e Sichieri (2002) observaram inadequações da Relação Cintura Quadril (RCQ) mais frequentemente entre indivíduos com menor escolaridade.

Acredita-se que conhecer os mecanismos que têm determinado a progressão acelerada da obesidade nos estratos menos favorecidos da população brasileira, certamente contribuirá para aumentar a efetividade das políticas e programas de controle desta epidemia (MONTEIRO; CONDE; CASTRO, 2003).

A prefeitura do município de Mauá-SP fornece hortifrútis por meio do Programa Banco de Alimentos para 17 instituições filantrópicas (creches, asilos e similares) que repassam os 
alimentos para famílias de baixa renda. Os Bancos de Alimentos (B.A's) são um programa do Governo Federal que realiza distribuição gratuita de frutas e hortaliças para entidades assistenciais, de forma a complementar as refeições diárias da população carente (MINISTÉRIO DO DESENVOLVIMENTO SOCIAL E COMBATE à FOME, 2005).

Com vistas a subsidiar o planejamento de ações de educação alimentar e nutricional, a serem desenvolvidas nas instituições, este estudo tem como objetivo identificar os alimentos considerados indispensáveis à alimentação saudável pela população carente segundo a escolaridade.

\section{Metodologia}

Trata-se de estudo transversal, descritivo, com coleta de dados primários, realizado nos meses de outubro a dezembro de 2012, em cinco instituições filantrópicas que recebem frutas, verduras e legumes do Programa Banco de Alimentos da Secretaria de Segurança Alimentar do município de Mauá-SP.

Os beneficiários foram convidados a comparecer às instituições nos dias previamente agendados para atividade de educação alimentar. Foram incluídas no estudo crianças com anuência dos pais e ciência do termo de assentimento, e adultos e idosos de ambos os sexos, mediante assinatura do termo de consentimento livre e esclarecido. Foram excluídas da amostra as crianças menores de sete anos, os adolescentes cujos responsáveis legais não compareceram para assinar o termo de consentimento livre e esclarecido $(n=5)$, quatro adultos por terem assinado o termo de consentimento livre e esclarecido, mas não terem participado da atividade proposta, e três adultos por não preencherem a ficha com dados sociodemográficos, apesar de terem assinado o termo e realizado a atividade. A pesquisa consistiu em atividade dinâmica, na qual foram disponibilizados recortes de alimentos retirados de folhetos de supermercado, sendo: frutas, verduras, legumes, arroz, feijão, leite, ovos, carnes (bovina, suína, de ave e peixe), empanado de frango, embutidos, sucos artificiais (garrafa e em pó), sucos à base de soja, refrigerantes, gelatina, iogurte, cereais matinais, achocolatado, margarina, maionese, requeijão, geleia, açúcar, extrato de tomate, tempero pronto, ketchup, salgadinho, biscoitos (recheados e salgados sem recheio), sopas e macarrão instantâneos. Os voluntários foram orientados a colar em folha sulfite os produtos que consideravam indispensáveis à alimentação saudável. Os alimentos correspondentes aos recortes foram quantificados em número de vezes que apareceram na folha da atividade proposta, sendo, portanto, os que foram considerados saudáveis pelos voluntários. Com intuito de traçar o perfil sociodemográfico da amostra estudada, foram coletadas, por meio de questionário autoaplicado, as seguintes informações: gênero, idade, renda familiar e escolaridade. As faixas de renda foram categorizadas segundo metodologia utilizada por Monticelli (2010). A classificação das faixas etárias foram crianças (até 11 anos de idade) e adolescentes (12 a 18 anos de idade), segundo o que preconiza o Estatuto da Criança e do Adolescente (BRASIL, 1990), e idosos (60 anos ou mais de idade), de acordo com o Estatuto do Idoso (BRASIL, 2003). Sendo assim, foram agrupados, na categoria adulto, os voluntários com idade entre 19 e 59 anos.

Nos casos de voluntários não alfabetizados ou com dificuldade visual para leitura, as respostas foram transcritas com o auxílio da responsável pela atividade.

\section{Análise estatística}

A análise estatística foi realizada no software Statistica 7.0, considerando o 
nível de significância $p<0,05$. As variáveis numéricas paramétricas foram descritas em média \pm desvio-padrão e as variáveis não paramétricas em mediana (mínimo - máximo). Aplicou-se o teste de quiquadrado para verificar possíveis associações entre as variáveis categóricas de estudo.

Esta pesquisa foi previamente aprovada pelo Comitê de Ética em Pesquisa (CEP) da Universidade Federal de São Paulo (UNIFESP), parecer número 103.461.

\section{Resultados}

\section{Caracterização da amostra}

A Tabela 1 demonstra as características biossociais dos voluntários que participaram do estudo. Aamostrafoicompostapor136indivíduos das diversas faixas etárias, sendo 101 adultos, 22 idosos e 13 crianças. Houve a participação de apenas dois adolescentes na atividade, o que representou $1 \%$ da amostra, por esse motivo, essa faixa etária não foi incluída no estudo.

Tabela 1. Características biossociais de beneficiários do Programa Banco de Alimentos da Prefeitura do Município de Mauá, São Paulo, 2012.

\begin{tabular}{lccccccccc}
\hline & \multicolumn{3}{c}{ Todos } & \multicolumn{2}{c}{ Crianças } & \multicolumn{2}{c}{ Adultos } & \multicolumn{2}{c}{ Idosos } \\
\cline { 2 - 10 } & $\mathrm{n}$ & $\%$ & $\mathrm{n}$ & $\%$ & $\mathrm{n}$ & $\%$ & $\mathrm{n}$ & $\%$ \\
\hline Faixa etária & 136 & 100 & 13 & 9,6 & 101 & 74,3 & 22 & 16,1 \\
\hline Gênero & 122 & 90 & 4 & 31 & 97 & 96 & 21 & 96 \\
\hline Feminino & 14 & 10 & 9 & 69 & 4 & 4 & 1 & 4 \\
Masculino & & & & & & & & \\
\hline Renda familiar & 80 & 59 & 6 & 46 & 62 & 61 & 12 & 55 \\
A - até R\$ 622,00 & 16 & 12 & 0 & 0 & 11 & 11 & 5 & 23 \\
B - de R\$ 622,00 a R\$ 1.244,00 & 10 & 7 & 1 & 8 & 8 & 8 & 1 & 5 \\
C - de R\$1.244,00 a R\$1.866,00 & 1 & 1 & 0 & 0 & 0 & 0 & 1 & 5 \\
D - de R\$ 1.866,00 a R\$ 2.488,00 & 2 & 1,5 & 0 & 0 & 2 & 2 & 0 & 0 \\
E - > R\$ 2.488,00 & 9 & 6,5 & 1 & 8 & 6 & 6 & 2 & 9 \\
F - Não possui renda & 18 & 13 & 5 & 38 & 12 & 12 & 1 & 5 \\
G - Não sabe & & & & & & & & \\
\hline Escolaridade & 12 & 9 & 0 & 0 & 8 & 8 & 4 & 18 \\
\hline A - Não estudou & 57 & 42 & 12 & 92 & 32 & 31 & 13 & 59 \\
B - 1 a 4án série & 26 & 19 & 1 & 8 & 21 & 21 & 4 & 18 \\
C - Ensino fundamental completo & 11 & 8 & 0 & 0 & 10 & 10 & 1 & 5 \\
D - Ensino médio incompleto & 27 & 20 & 0 & 0 & 27 & 27 & 0 & 0 \\
E - Ensino médio completo & 1 & 1 & 0 & 0 & 1 & 1 & 0 & 0 \\
F - Ensino superior incompleto & 1 & 1 & 0 & 0 & 1 & 1 & 0 & 0 \\
G - Ensino superior completo & 1 & 1 & 0 & 0 & 1 & 1 & 0 & 0 \\
H - Não sabe & & & & & & &
\end{tabular}

n: número de voluntários Fonte: As autoras.
$\%=$ frequência percentual 
Observou-se que 90\% dos voluntários da amostra total correspondiam ao gênero feminino $(n=122)$. Em relação à renda, a maior parte dos voluntários declarou ter renda familiar de até $\mathrm{R} \$ 622,00$, não havendo grandes diferenças entre as faixas etárias. Em relação ao nível de escolaridade, a maior parte declarou ter completado apenas o $4 \underline{\mathrm{o}}$ ano do ensino fundamental (42\%) e apenas 20\% completou o ensino médio.

\section{Alimentos considerados indispensáveis à alimentação saudável}

ATabela2demonstraosalimentosqueforam incluídos pelos voluntários da atividade realizada, como indispensáveis à alimentação saudável.

Tabela 2 - Alimentos considerados indispensáveis à alimentação saudável pelos beneficiários do Programa Banco de Alimentos da Prefeitura do Município de Mauá, São Paulo, 2012.

\begin{tabular}{|c|c|c|}
\hline Alimento & $\begin{array}{c}\mathrm{N}^{\circ} \text { de } \\
\text { voluntários que } \\
\text { considerou }\end{array}$ & $\%$ \\
\hline Achocolatado & 32 & 23 \\
\hline Açúcar & 46 & 34 \\
\hline Arroz & 111 & 81 \\
\hline Bebida de soja & 79 & 58 \\
\hline Biscoito recheado & 33 & 24 \\
\hline Biscoito salgado & 59 & 43 \\
\hline Carne & 119 & 87 \\
\hline Catchup & 12 & 9 \\
\hline Cereal matinal & 50 & 36 \\
\hline Embutido & 45 & 33 \\
\hline Empanado de frango & 23 & 17 \\
\hline Extrato de tomate & 48 & 35 \\
\hline Feijão & 107 & 78 \\
\hline Gelatina & 91 & 67 \\
\hline Geleia de fruta & 42 & 30 \\
\hline Hortifrútis & 135 & 99 \\
\hline logurte & 95 & 70 \\
\hline Leite & 109 & 80 \\
\hline Macarrão instantâneo & 25 & 18 \\
\hline Maionese & 39 & 28 \\
\hline Margarina & 59 & 43 \\
\hline Ovo & 94 & 69 \\
\hline
\end{tabular}




\begin{tabular}{lll}
\hline Refrigerante & 28 & 20 \\
Requeijão & 51 & 37 \\
Salgadinho & 17 & 12 \\
Sopa instantânea & 53 & 39 \\
Suco artificial & 66 & 48 \\
Tempero pronto & 34 & 25 \\
\hline
\end{tabular}

Fonte: As autoras.

$\%=$ frequência percentual

Os alimentos mais citados como $(p=0,002)$, embutidos $(p=0,001)$, empanado sendo indispensáveis à alimentação saudável de frango $(p=0,001)$, cereal matinal (por mais de 65\% dos voluntários) foram o $(p=0,010)$, biscoito recheado $(p=0,001)$, grupo das frutas, verduras e legumes, carnes, sopa instantânea $(p=0,000)$, maionese arroz, leite, feijão, iogurte, ovo e gelatina. $(p=0,001)$, extrato de tomate $(0,002)$,

No entanto, aproximadamente metade macarrão instantâneo $(p=0,0012)$ e tempero da amostra considerou indispensável o pronto $(p=0,001)$, conforme Figura 1. consumo de suco artificial (48\%) e 25\% a adição de temperos prontos industrializados. Mais de $30 \%$ da amostra incluiu embutidos e açúcar.

Em relação à escolaridade, foi possível observar associações significativas com a citação de produtos como: suco artificial É importante pontuar que as categorias de ensino superior completo, superior incompleto e aqueles que não souberam informar o nível de escolaridade foram categorias excluídas das análises, pois apresentavam apenas um voluntário em cada categoria.

Figura 1 - Associação entre escolaridade e porcentagem de citação de alimentos como saudáveis.

Suco Artificial

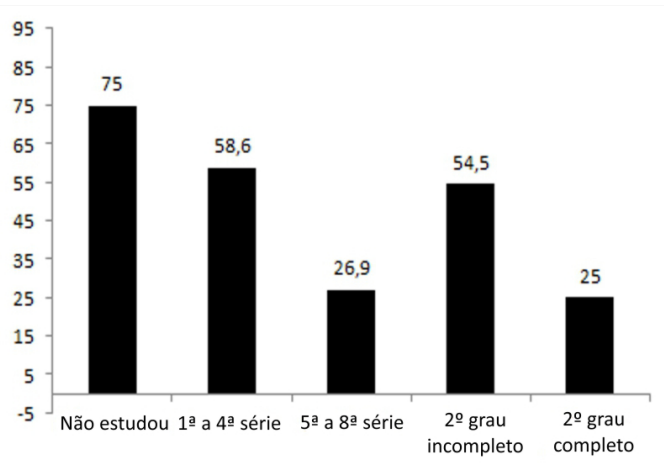

Embutidos

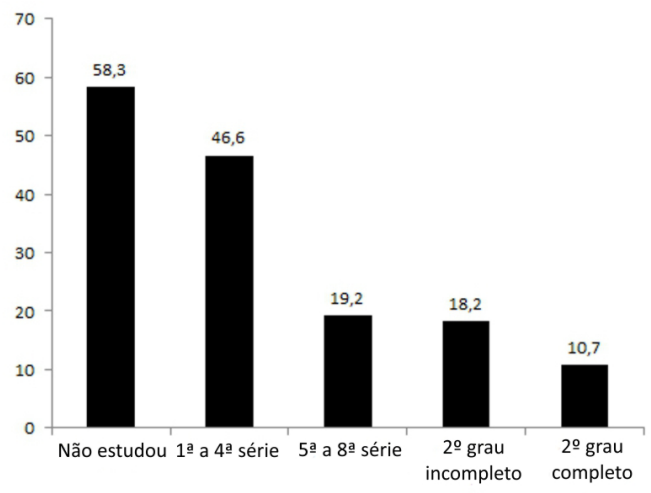


Empanado de Frango

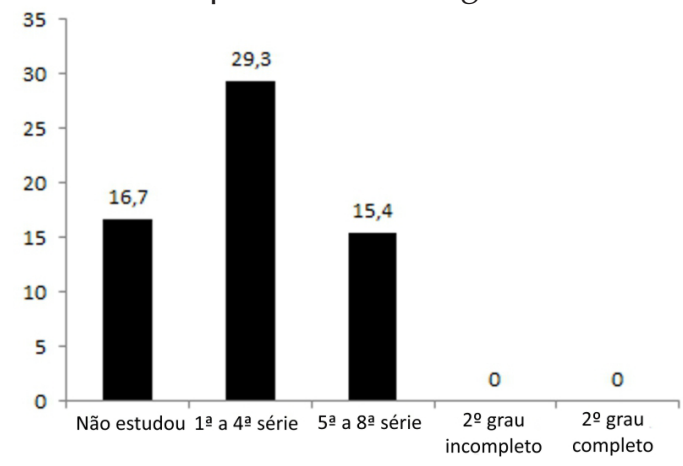

Biscoito Recheado

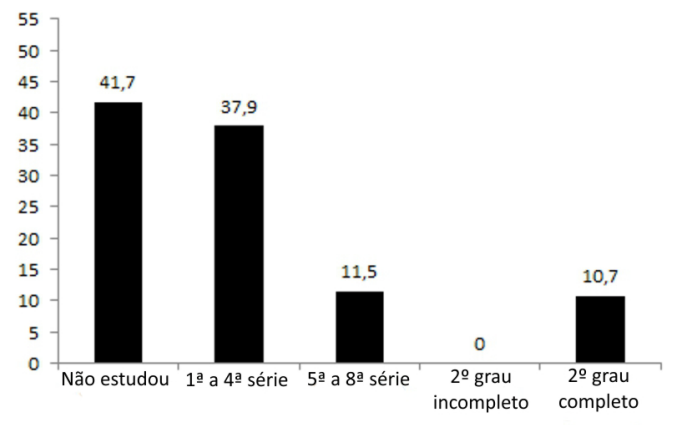

Maionese

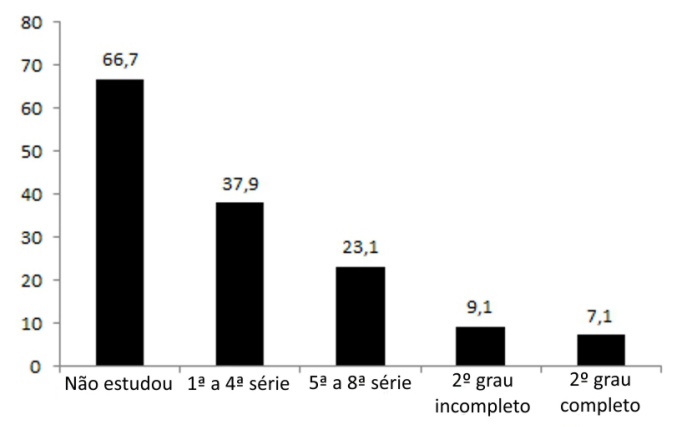

Macarrão Instantâneo

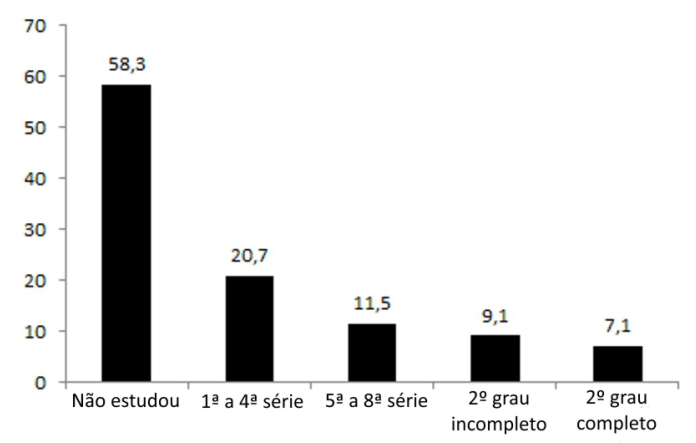

$* \mathrm{p}<0,05 \quad \%$ : Frequência percentual.
Cereal Matinal

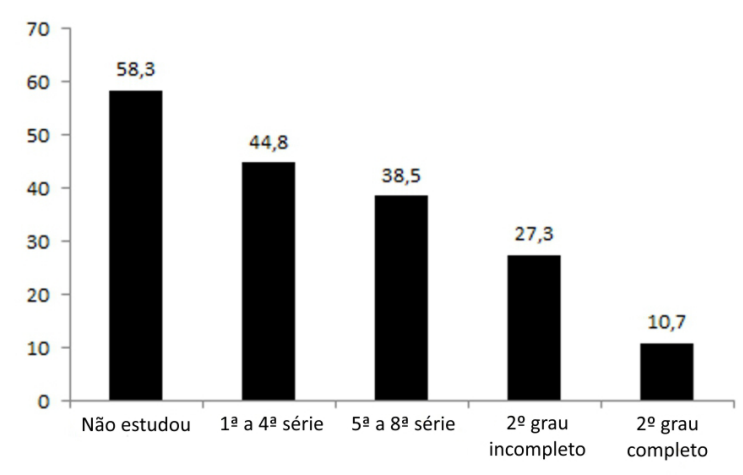

Sopa Instantânea

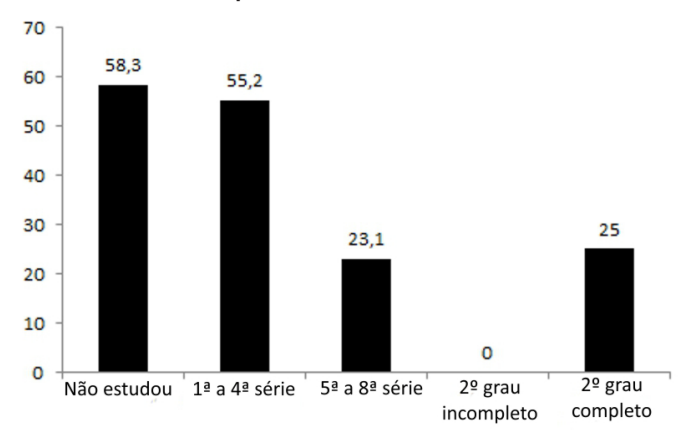

Extrato de Tomate

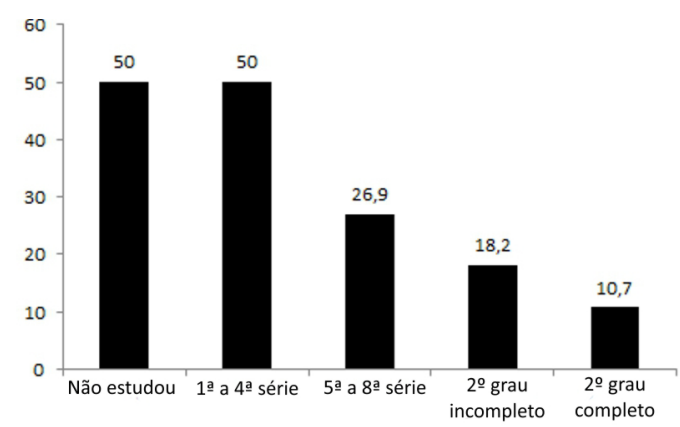

Tempero Pronto

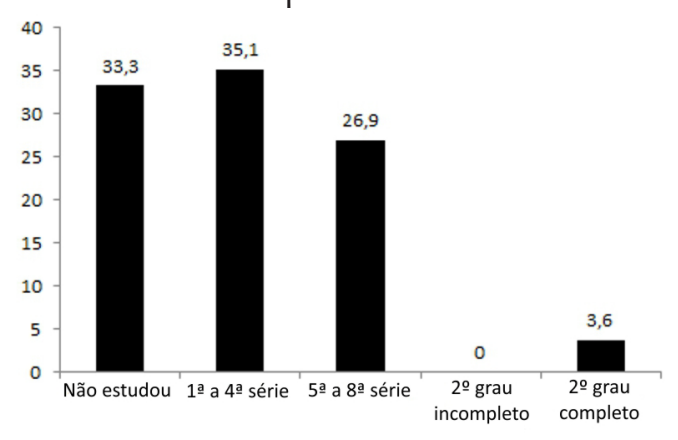

Fonte: As autoras. 
Na Figura 1, nota-se que, para as variáveis "embutidos", "cereal matinal", "maionese", "extrato de tomate" e "macarrão instantâneo", a porcentagem de citação reduz conforme aumenta o nível de escolaridade.

Nacategoriadepessoasquenãoestudaram, notou-se associação positiva com a percepção do suco artificial, embutidos, maionese e macarrão instantâneo como alimentos saudáveis. Entre aqueles que estudam ou estudaram da $1 \underline{a}$ a $4 \underline{a}$ série, observou-se também associação positiva com a citação do suco artificial, embutidos, empanado de frango, biscoito recheado, sopa instantânea, maionese, extrato de tomate e tempero pronto como produtos saudáveis.

A categoria de pessoas que cursaram o $2^{\text {o }}$ grau completo se contrapõe a inclusão de suco artificial, embutidos, empanado de frango, cereal matinal, maionese, extrato de tomate e tempero pronto como alimentos indispensáveis à saúde. Já a categoria de $2^{2}$ grau incompleto, não percebe o biscoito recheado, a sopa instantânea e o tempero pronto como alimentos saudáveis. Entre aqueles que estudaram de $5^{\underline{a}}$ a 8 a série, notou-se apenas associação negativa do suco artificial como produto saudável.

\section{Discussão}

A presente investigação verificou que indivíduos com menor escolaridade consideram indispensáveis produtos de baixo valor nutritivo e com elevadas quantidades de açúcar, gordura e/ ou sódio, como: suco artificial, biscoito recheado, cereal matinal, empanado de frango, maionese, embutidos, extrato de tomate, macarrão instantâneo, sopa instantânea e tempero pronto.

A consideração de biscoito recheado e empanado de frango como alimentos saudáveis é preocupante, uma vez que estes produtos possuem gordura trans em sua composição. O consumo desse tipo de gordura está relacionado à elevação do colesterol de baixa densidade LDL (Low Density Lipoprotein), redução do colesterol de alta densidade HDL (High Density Lipoprotein) e, principalmente, ao desenvolvimento de doenças coronarianas (BERTOLINO et al., 2006). Adicionalmente, o biscoito recheado possui elevado teor de açúcar simples, queelevam mais rapidamentea glicemia.

A citação de suco artificial como sendo saudável está em consonância com o aumento do consumo deste produto pela população, que demonstra a tendência de procurar, muitas vezes de forma equivocada, alimentos saudáveis que oferecem conveniência (DE MARCHI, 2001). No entanto, sucos artificiais são veículos importantes de açúcar, o que representa uma fonte adicional de calorias (NOGUEIRA; SICHIERI, 2009). Estudos mostraram associação positiva entre o consumo de sucos industrializados e o aumento do Índice de Massa Corporal (IMC) (NOGUEIRA; SICHIERI, 2009; SANIGORSKI; BELL; SWINBURN, 2007; TANASESCU et al., 2000). Drewnowski e outros (2007) sugerem que o elevado consumo de bebidas açucaradas decorra da propaganda indiscriminada destes produtos que atinge frequentemente os domicílios. Pesquisa realizada com 1530 voluntários (BARROS, 2008) verificou que, entre os alimentos industrializados mais consumidos por adultos e idosos no município de São Paulo, estavam: manteiga e margarina, refrigerante, suco artificial, embutidos, hambúrgueres, empanado de frango e molhos à base de maionese. Esses resultados condizem com os encontrados nesta pesquisa, uma vez que indivíduos com menor escolaridade demonstraram considerar indispensável o consumo de suco artificial, embutidos, empanado de frango e maionese. Em contrapartida, naquele estudo, foi verificado maior consumo de alimentos industrializados por indivíduos de maior 
nível socioeconômico. Vale ressaltar que a presente pesquisa não avaliou o consumo, e sim a consideração dos produtos com sendo saudáveis. Desta forma, pode-se sugerir possível valorização dos alimentos consumidos por indivíduos de maior nível socioeconômico por parte dos voluntários de menor escolaridade.

Alimentos com elevados teores de sódio, como embutidos, macarrão instantâneo e temperos prontos, também foram mais citados por indivíduos de menor escolaridade (Figura 1). Molina e outros (2003) verificaram que a ingestão de sal e produtos com alto teor desse mineral foi maior entre indivíduos de menor nível socioeconômico, visto que alimentos preparados com temperos prontos são bastante acessíveis a esse público, podendo explicar, em parte, a alta prevalência de hipertensão arterial nas classes socioeconômicas mais baixas. Além disso, a associação entre baixa escolaridade e maior consumo de alimentos de risco para doença cardiovascular (fontes de gorduras saturadas, sódio e açúcares) em adultos foi encontrada no trabalho de Neumann, Shirassu e Fisberg (2006).

Brito et al. (2012) estudaram o nível de conhecimento em nutrição de duas comunidades de risco social, residentes no Ceará - Brasil, e verificaram alto índice de erro em diversos aspectos investigados sobre o tema. Os autores acreditam que tal constatação pode ser justificada pelo baixo nível de escolaridade dos chefes das famílias investigadas, quase metade possui o Ensino Fundamental incompleto, o mesmo dos voluntários deste estudo (Tabela 1).

No presente estudo, outro alimento com elevado teor de sódio, citado principalmente por indivíduos com estudos entre 1a e a $4^{\underline{a}}$ série, foi a sopa instantânea. Costa (2010) analisou o anúncio de uma determinada marca de sopas industrializadas e observou que a propaganda se apropria do conhecimento técnico-científico para estimular o consumo do produto. A embalagem apresenta como imagem de fundo, vegetais de cor acentuada, que "transpiram" água na intenção de ressaltar a ideia de alimentos naturais e frescos.

Sabe-se que a publicidade tem explorado o conhecimento científico nos discursos de anúncios de alimentos, supervalorizando ou mistificando uma ou mais de suas características (COSTA, 2010), o que pode ter contribuído para a consideração de alimentos processados ricos em açúcar, gordura e/ou sódio como saudáveis.

Diante das análises e exposições, concluímos que indivíduos menos escolarizados demonstraram menor conhecimento sobre alimentação saudável, mesmo se tratando de amostra na qual o nível de escolaridade total é baixo. Isso sugere que poucos anos de estudo podem fazer diferença no entendimento sobre saúde e alimentação.

\section{Considerações finais}

Os beneficiários do Programa Banco de Alimentos da prefeitura do município de Mauá demonstraram acreditar que frutas, verduras e legumes, carnes, arroz, leite e feijão são alimentos saudáveis. No entanto, a categoria de indivíduos com menor escolaridade associou-se positivamente à citação de produtos processados ricos em açúcar, gordura e/ou sódio como sendo indispensáveis à alimentação saudável, tais como: suco artificial, biscoito recheado, cereal matinal, empanado de frango, maionese, embutidos, extrato de tomate, macarrão instantâneo,sopainstantâneaetemperosprontos.

Tais dados sugerem que a população menos escolarizada pode estar mais suscetível a interpretações errôneas sobre conceitos de alimentação e nutrição e alertam para a necessidade de programas de educação alimentar voltadas para esse público. Julga-se apropriada também a realização de pesquisas acerca da influência dos meios de comunicação na concepção sobre alimentação saudável. 


\section{Agradecimentos}

Às estudantes de Nutrição da Faculdade de Mauá (FAMA), Márcia Cristina de Godoy Alves e Taide Helena Held, pelo auxílio na coleta de dados; e ao Sr. Marco Filório, Secretário de Segurança Alimentar, que permitiu a realização deste trabalho.

\section{Referências}

BARROS, R. R. Consumo de alimentos industrializados e fatores associados em adultos e idosos residentes no município de São Paulo. 2008. 172f. Dissertação (Mestrado) - Faculdade de Saúde Pública/Universidade de São Paulo, São Paulo, 2008.

BRASIL. Estatuto da criança e do adolescente. Lei no 8.069, de 13 de julho de 1990. Dispõe sobre o Estatuto da Criança e do Adolescente e dá outras providências. Brasília, DF, 1990. Disponível em: <http://www.planalto.gov.br/ccivil_03/leis/L8069.htm>. Acesso em: 20 fev. 2013.

Estatuto do idoso. Lei no 10.741, de 01 de outubro de 2003. Dispõe sobre o Estatuto do Idoso e dá outras providências. Brasília, DF: Senado Federal, 2003. Disponível em: <http://www. planalto.gov.br/ccivil_03/leis/2003/L10.741.htm >. Acesso em: 20 fev. 2013.

. Ministério da Saúde. Secretaria de Vigilância em Saúde. VIGITEL Brasil, 2012: vigilância de fatores de risco e proteção para doenças crônicas por inquérito telefônico. Brasília: Ministério da Saúde, 2012.

BERTOLINO, C. N. et al. Influência do consumo alimentar de ácidos graxos trans no perfil de lipídios séricos em nipo-brasileiros de Bauru, São Paulo, Brasil. Cadernos de Saúde Pública, Rio de Janeiro, v. 22, n. 2, p. 357-64, fev. 2006.

BRITO, C. B. R. et al. Inter-relação entre as práticas alimentares e o grau de conhecimento em nutrição das famílias residentes em duas comunidades de risco em Fortaleza, CE. Revista de APS, Juiz de Fora, v.15, n.1, p. 36-41, jan./mar. 2012.

CASTRO, N. M. G.; DÁTTILO, M; LOPES, L. C. C. Avaliação do conhecimento nutricional de mulheres fisicamente ativas e sua associação com o estado nutricional. Revista Brasileira de Ciências do Esporte, Campinas, v. 32, n.1, p. 161-172, set. 2010.

COSTA, M. F. Consumo alimentar: discurso científico em anúncios publicitários. Contemporânea, Rio de Janeiro, v. 7, n. 3, p. 130-140, 2010.

DATTILO, M. et al. Conhecimento nutricional e sua associação com o índice de massa corporal. Nutrire, São Paulo, v. 34, n. 1, p. 75-84, abr. 2009.

DE MARCHI, R. Desenvolvimento de uma bebida a base de maracujá (Passiflora edulis Sims. F. flavicarpa Deg.) com propriedades de reposição hidrolítica. 2001. 92f. Dissertação (Mestrado) - Faculdade de Ciências Farmacêuticas, Universidade Estadual Paulista "Julio de Mesquita Filho", Araraquara. 2001. 
DREWNOWSKI, A. et al. Low-energy-density diets are associated with higher diet quality and higher diet costs in French adults. Journal of the American Dietetic Association, Toronto, v. 107, n. 6, p. 1028-1032, 2007.

GIUGLIANO, R.; CARNEIRO, E. C. Fatores associados à obesidade em escolares. Jornal de Pediatria, Rio de Janeiro, v. 80, n. 1, p. 17-22, jan./fev. 2004.

GUTIERREZ-FISAC, J. L. et al. The size of obesity differences associated with educational level in Spain, 1987-1995/97. Journal Epidemiology and Community Health, London, v. 56, n. 6, p. 457460, 2002.

MACHADO, P. A. N.; SICHIERI, R. Relação cintura-quadril e fatores de dieta em adultos. Revista de Saúde Pública, São Paulo, v. 36, n. 2, p. 198-204, 2002.

MINISTÉRIO DO DESENVOLVIMENTO SOCIAL E COMBATE à FOME (MDS). Roteiro de implantação para bancos de alimento. 2005. Disponível em: <http://www.mds.gov.br/backup/ editais/san2009/editais_e_outros/roteiro_de_implantacao_-_banco_de_alimentos.pdf >. Acesso em: 20 jun. 2012.

MOLARIUS, A. et al. Educational level, relative body weight, and changes in their association over 10 years: an international perspective from the WHO MONICA Project. American Journal of Public Health, Bethesda, v. 90, n. 8, p. 1260-1268, 2000.

MOLINA, M. del C. B. et al. Hipertensão arterial e consumo de sal em população urbana. Revista de Saúde Pública, São Paulo, v. 37, n. 6, p. 743-50, 2003.

MONTEIRO, C. A.; CONDE, W. L.; CASTRO, I. R. R. A tendência cambiante da relação entre escolaridade e risco de obesidade no Brasil (1975-1997). Cadernos de Saúde Pública, Rio de Janeiro, v. 19, suppl.1, 2003.

MONTEIRO, C. A.; CONDE, W. L.; POPKIN, B. M. Independent effects of income and education on the risk of obesity in the Brazilian adult population. The Journal of nutrition. Bethesda, v. 131, n. 3, p. 881S-886S, 2001.

MONTICELLI, F. D. B. Consumo alimentar de adolescentes de escolas da rede municipal de ensino da cidade de Curitiba. Dissertação (Mestrado em Nutrição em Saúde Pública) - Faculdade de Saúde Pública, Universidade de São Paulo, São Paulo, 2010.

NEUMANN, A. I. de L. C. P.; SHIRASSU, M. M.; FISBERG, R. M. Consumo de alimentos de risco e proteção para doenças cardiovasculares entre funcionários públicos. Revista de Nutrição, Campinas, v. 19, n. 1, p. 19-28, jan./fev. 2006.

NOGUEIRA, F. de A. M.; SICHIERI, R. Associação entre consumo de refrigerantes, sucos e leite, com o índice de massa corporal em escolares da rede pública de Niterói. Cadernos de Saúde Pública, Rio de Janeiro, v. 25, n. 12, p. 2715-2724, dez. 2009.

SANIGORSKI, A. M.; BELL, A. C.; SWINBURN, B. A. Association of key foods and beverages with obesity in Australian schoolchildren. Public Health Nutrition, Massachusetts, v. 10, n. 2, p. 152 $157,2007$. 
TANASESCU, M. et al. Biobehavioral factors are associated with obesity in Puerto Rican children. The Journal of Nutrition, Bethesda, v. 130, n. 7, p. 1734-1742, 2000.

TARDIDO, A. P.; FALCÃO, M. C. O impacto da modernização na transição nutricional e obesidade. Revista Brasileira de Nutrição Clínica, São Paulo, v. 21, n. 2, p. 117-24, 2006.

TEICHMANN, L. et al. Fatores de risco associados ao sobrepeso e a obesidade em mulheres de São Leopoldo, RS. Revista Brasileira de Epidemiologia, São Paulo, v. 9, n. 3, p. 360-373, set. 2006.

TORRANCE, G. M.; HOOPER, M. D.; REEDER, B. A. Trends in overweight and obesity among adults in Canada (1970-1992): evidence from national surveys using measured height and weight. International Journal of Obesity, London, v. 26, n. 6, p. 797-804, 2002.

VELÁSQUEZ-MELÉNDEZ, G.; PIMENTA, A. M.; KAC, G. Epidemiologia do sobrepeso e da obesidade e seus fatores determinantes em Belo Horizonte (MG), Brasil: estudo transversal de base populacional. Revista Panamericana de Salud Pública / Pan American Journal of Public Health, Washington, v.16, n. 5, p. 308-314, 2004.

WANG, Y.; MONTEIRO, C.; POPKIN, B. M. Trend of obesity and underweight in older children and adolescents in the USA, Brazil, China and Russia. American Journal of Clinical Nutrition, Bethesda, v. 75, n. 6, p. 971-77, jun. 2002.

WARDLE, J.; PARMENTER, K.; WALLER, J. Nutrition knowledge and food intake. Appetite, v. 34, n. 3, p. 269-275, jun. 2000.

WARDLE, J.; WALLER, J.; JARVIS, M. J. Sex differences in the association of socioeconomic status with obesity. American Journal of Public Health, Bethesda, v. 92, n. 8, p. 1299-1304, 2002.

Submetido em 25 de outubro de 2013.

Aprovado em 7 de março de 2014. 